\title{
Survival and complication free survival in Marfan's syndrome: implications of current guidelines
}

M Groenink, T A J Lohuis, J G P Tijssen, M S J Naeff, R C M Hennekam, E E van der Wall, B J M Mulder

\begin{abstract}
Objective-To evaluate survival and complication free survival in patients with Marfan's syndrome and to assess the possible influence of recently revised guidelines for prophylactic aortic root replacement in these patients.

Methods-130 patients who had been attending one institution over 14 years were evaluated. Kaplan-Meier analysis was performed in 125 patients who did not present with aortic root dissection as the first sign of Marfan's syndrome, with the end points: death, aortic root dissection, and prophylactic aortic root replacement after diagnosis. In the patients developing aortic root dissection, current guidelines for prophylactic aortic root replacement were retrospectively applied to investigate the number of dissections that could theoretically have been prevented. The guidelines were: (1) aortic root diameter $\geqslant 55 \mathrm{~mm}$, (2) positive family history of aortic dissections and aortic root diameter $\geqslant 50$ $\mathrm{mm}$, and (3) aortic root growth $\geqslant 2$ $\mathrm{mm} /$ year. Outcomes following emergency surgery (15 patients) and prophylactic surgery of the aortic root (30 patients) were compared.
\end{abstract}

Results-Five and 10 year survival after diagnosis was $95 \%$ and $88 \%$, and the five and 10 year complication free survival was $78 \%$ and $66 \%$, respectively. Thirteen patients developed dissection, 30 underwent prophylactic repair, and 82 had an uncomplicated course. Eleven dissections could theoretically have been prevented by application of the current guidelines. Five year survival following emergency and prophylactic repair of the aortic root was $51 \%$, and $97 \%$, respectively.

Conclusions-Survival in the Marfan's syndrome in the past 14 years seems satisfactory; with application of current guidelines, it has probably even improved. However, because of the high fatality rate in Marfan patients developing aortic root dissection, more extensive screening for Marfan's syndrome and a search for additional risk factors are desirable. (Heart 1999;82:499-504)

Keywords: Marfan's syndrome; aortic root dissection; survival
Prognosis in patients with Marfan's syndrome is mainly determined by aortic complications, especially of the aortic root. ${ }^{1-5}$ Clinical management is primarily aimed at the prevention of dissection of aortic root aneurysms, as these complications are associated with a high mortality. ${ }^{6-8}$ Improvements in surgical techniques during recent decades have facilitated the prophylactic replacement of the aortic root with a composite graft (the Bentall procedure). ${ }^{7-10}$ Application of this technique was probably the main reason for the increase in survival from 40 years in 1970 to approximately 60 years in $1990 .^{35}$ Prophylactic replacement of the aortic root was originally performed when it had reached a diameter of $60 \mathrm{~mm}$ (previous guidelines). ${ }^{11-13}$ Recently, new guidelines have been developed and prophylactic surgery is now recommended at an aortic root diameter of $\geqslant 55 \mathrm{~mm}$ in adults, at an aortic root diameter $\geqslant 50 \mathrm{~mm}$ in children, and in adults with a family history of aortic dissection, and when excessive aortic root growth occurs (current guidelines). ${ }^{13-17}$ In only one study has excessive aortic root growth been quantitatively defined as an annual increase in aortic root ratio of $\geqslant 5 \%$, representing approximately $2 \mathrm{~mm}$ /year in adults with Marfan's syndrome. ${ }^{17}$ In children with Marfan's syndrome, excessive aortic root growth is even more difficult to quantify because of concomitant physiological aortic root growth. ${ }^{18-23}$

The influence of revised guidelines on survival and complication free survival is still unknown. Accordingly, our aims in this study were first, to determine survival and complication free survival in the patients who were diagnosed as having Marfan's syndrome in our institution between 1984 and 1998; second, to assess the possible influence of current guidelines for prophylactic repair, when applied retrospectively to the patients who developed aortic root dissection in the period from 1984 to 1998; third, to investigate possible differences in Marfan related features and aortic root growth between patients without complications, prophylactically operated patients, and patients who developed aortic root dissection; and finally, to determine differences in survival between patients who were operated prophylactically and patients who underwent emergency surgery for aortic root dissection.

\section{Methods}

PATIENT POPULATION

From 1984 until 1998, 130 patients were diagnosed as having Marfan's syndrome by the
Accepted for publication 13 April 1999 
Table 1 Clinical characteristics of 125 patients with Marfan's syndrome at time of diagnosis, selected by aortic root complications

\begin{tabular}{lllll}
\hline & $\begin{array}{l}\text { Total } \\
(n=125)\end{array}$ & $\begin{array}{l}\text { No aortic root } \\
\text { complications }(A, n=82)\end{array}$ & $\begin{array}{l}\text { Elective aortic root } \\
\text { replacement }(B, n=30)\end{array}$ & $\begin{array}{l}\text { Aortic root dissection } \\
(C, n=13)\end{array}$ \\
\hline Sex (M/F) (mean (SD)) & $64 / 61$ & $37 / 45$ & $20 / 10$ & $7 / 6$ \\
Age (years) (m) & $21(15)$ & $19(15)$ & $24(14)$ & $30(15)$ \\
$\begin{array}{l}\text { Aortic root diameter (mm) (mean (SD)) } \\
\text { Positive family history of }\end{array}$ & $38(12)$ & $33(9)$ & $49(15)$ & $42(8)$ \\
$\quad \begin{array}{l}\text { Marfan's syndrome } \\
\text { Dissection }\end{array}$ & $71 \%$ & $72 \%$ & $67 \%$ & $77 \%$ \\
\hline
\end{tabular}

multidisciplinary screening team of our institution. Diagnosis was performed according to the Berlin criteria during the period 1986-1996 and according to the revised Ghent criteria thereafter. ${ }^{24}{ }^{25}$ Of the 130 patients, five presented with aortic root dissection as the first symptom of Marfan's syndrome and they were subsequently excluded from follow up analysis. Survival and complication free survival of the remaining 125 patients were assessed with well defined end points - death, death or aortic root dissection, and death or aortic root dissection or prophylactic replacement of the aortic root.

RISK ASSESSMENT

For risk assessment with respect to aortic root complications, the 125 patients were divided into three groups:

Group A: no aortic root dissection or aortic root surgery ( $\mathrm{n}=82$, age range 0 to 68 years); Group B: prophylactic replacement of the aortic root ( $\mathrm{n}=30$, age range 3 to 49 years);

Group C: dissection of the aortic root $(n=13$, age range 11 to 55 years).

Table 1 shows the clinical characteristics of the patients. All aortic root diameters were measured by $\mathrm{M}$ mode or cross sectional echocardiography, which can be used interchangeably as we have shown in an earlier study ${ }^{26}$ The occurrence of Marfan related cardiovascular, ocular, skeletal, integumentary, and pulmonary features, the presence of affected family members with or without aortic dissection, and annual aortic root growth rate were compared in the three groups. Annual aortic root growth was assessed in 85 patients, who were older than 16 years of age, and in whom at least two sequential measurements of aortic root diameter were available. In the group without aortic complications (group $\mathrm{A}, \mathrm{n}=52$ ), the most recent measurements were analysed, whereas in the prophylactically operated group (group $\mathrm{B}, \mathrm{n}=21$ ) and in the group of patients who developed aortic root dissection (group C, $\mathrm{n}=12$ ) the final measurements before surgery or dissection were analysed. Aortic root growth was expressed as the slope of the linear correlation between aortic root size and age.

\section{CURRENT GUIDELINES}

The patients who developed aortic root dissection were retrospectively analysed in relation to the current guidelines for prophylactic replacement of the aortic root-that is, aortic root diameter $\geqslant 55 \mathrm{~mm}$; aortic root diameter $\geqslant 50$ $\mathrm{mm}$ and a family history of aortic dissection; or growth of the aortic root diameter of $\geqslant 2$ $\mathrm{mm} /$ year
We subsequently investigated how the current guidelines might hypothetically have prevented aortic root dissection in these patients.

COMPARISON OF SURVIVAL AFTER PROPHYLACTIC AND EMERGENCY SURGERY

Survival of the patients who underwent prophylactic replacement and emergency repair of the aortic root (by means of the button Bentall procedure ${ }^{27}$ ) was compared. The five patients who presented with aortic root dissection as the first symptom of Marfan's syndrome were reincluded only for this analysis $(n=130)$.

STATISTICAL ANALYSIS

Survival and event free survival were calculated by Kaplan-Meier analysis. The occurrence of Marfan related features was compared in the three groups by the Fisher exact probability test. Differences in aortic root growth rates between the three groups were assessed using the Mann-Whitney U test. A probability (p) value of $<0.05$ was considered significant.

\section{Results}

SURVIVAL AND EVENT FREE SURVIVAL

Mean (SD) follow up of the 125 patients was 8.9 (4.0) years. Eleven patients died, all as a result of cardiovascular complications, and nine $(82 \%)$ as a direct result of aortic root dissection. Cumulative survival and complication free survival during 16 years were assessed by Kaplan-Meier analysis (fig 1). Five year and 10 year cumulative survival was $95 \%$ and $88 \%$, respectively. After five and 10 years, the complication free survival was $78 \%$ and $66 \%$, respectively.

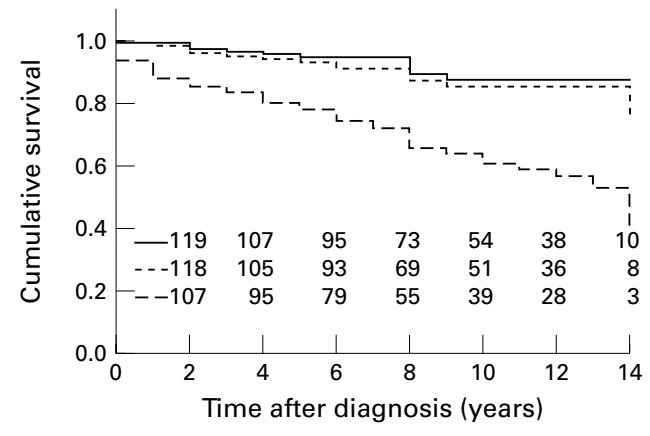

Figure 1 Kaplan-Meier curves of survival

(uninterrupted line), survival without aortic root dissection (dotted line), and survival without aortic root complications (dashed line) in 125 Marfan patients. The numbers of patients followed up are given in the lower part of the figure. 


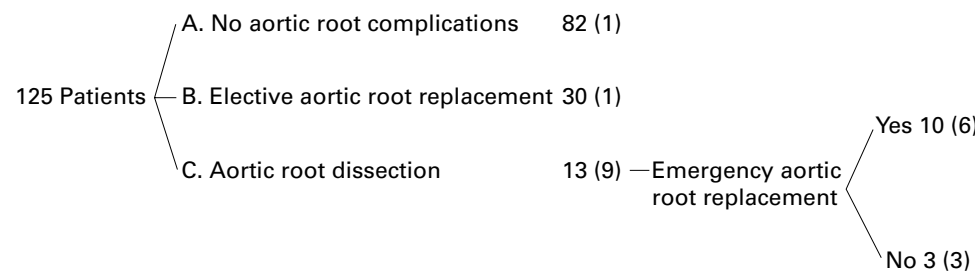

Figure 2 Subdivision of 125 patients into the three groups $A, B$, and $C$, according to aortic root complication. Absolute numbers are indicated, with deceased patients in brackets.

RISK ASSESSMENT

For risk analysis, the patients were divided into three groups. Figure 2 shows the number of patients in the three groups schematically.

Of the 82 patients in whom no aortic root complication occurred (66\%), one patient died of heart failure owing to severe mitral regurgitation at the age of 17 months and one patient developed type B dissection at 47 years.

Thirty patients $(24 \%)$ underwent prophylactic aortic root surgery. One patient in this group died of cardiac arrest postoperatively at the age of 12 years, three patients developed aortic complications at other levels of the aorta, and in one patient reoperation on the aortic root was necessary.

Of the 13 patients who developed aortic root dissection $(10 \%)$, three died before reaching hospital. Five of the remaining 10 patients died perioperatively and one patient died six years after emergency surgery from rupture of an abdominal aortic aneurysm. Two patients developed type B dissection before aortic root dissection occurred. Of the five patients who survived emergency surgery, four developed aneurysms in the aortic arch or the descending aorta, in two cases leading to further surgical intervention.

The occurrence of Marfan related clinical features was compared between the three groups. In patients without aortic root complications (group A) and in those with prophylactic repair (group B), lens subluxation was present in $45 \%(37 / 82)$ and $53 \%(16 / 30)$, respectively. In patients with aortic root dissection (group C), there was significantly less lens subluxation (1/13; $\mathrm{p}<0.05 v$ group $\mathrm{A}$; $\mathrm{p}<0.01 v$ group B). No significant differences in other Marfan related phenotypic features or in familial involvement could be demonstrated between the three groups.

Table 2 shows the clinical characteristics of the 43 patients who underwent aortic root surgery. Prophylactic aortic root replacement was performed at a mean age of 28 years and at a mean aortic root diameter of $60 \mathrm{~mm}$, whereas aortic dissection developed at a mean age of 35 years and at a mean aortic root diameter of 53 $\mathrm{mm}$.

Table 2 Clinical characteristics in 43 patients with Marfan's syndrome at time of elective aortic root replacement or aortic root dissection

\begin{tabular}{llll}
\hline & Total $(n=43)$ & $\begin{array}{l}\text { Elective aortic root } \\
\text { replacement }(B, n=30)\end{array}$ & $\begin{array}{l}\text { Aortic root dissection } \\
(C, n=13)\end{array}$ \\
\hline Age (years) & $30(13)$ & $28(12)$ & $35(14)$ \\
Aortic root diameter (mm) & $58(11)$ & $60(12)$ & $53(7)$ \\
Time since diagnosis (years) & $4.7(4.0)$ & $4.5(4.2)$ & $5.3(3.7)$ \\
Aortic root growth (mm/year) & $2.9(3.4)$ & $3.6(4.0)$ & $1.8(1.3)$ \\
\hline
\end{tabular}

Values are mean (SD)

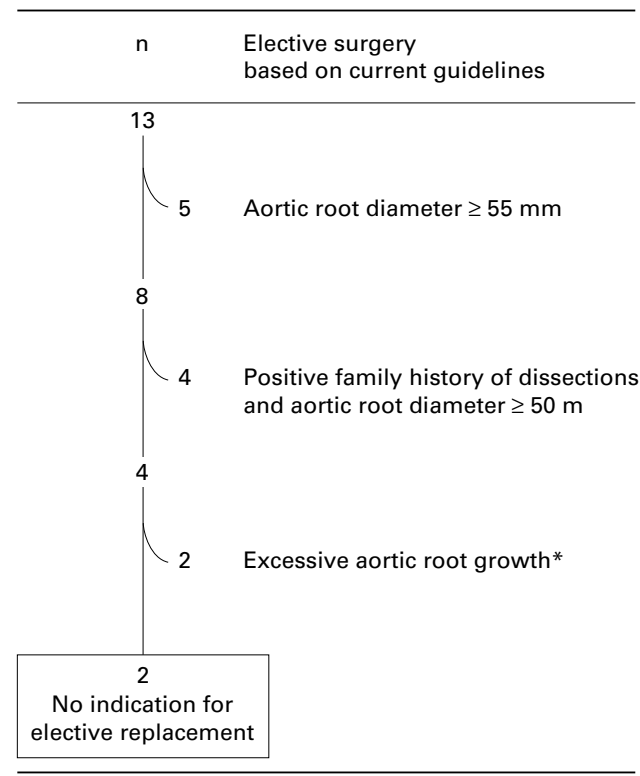

Figure 3 Hypothetical clinical course of 13 patients with Marfan's syndrome who developed aortic root dissection in the previous 14 years, when current guidelines were retrospectively applied. $n$, number of patients; *annual aortic root growth rate of $>2 \mathrm{~mm} /$ year. In 11 of the 13 patients, current guidelines would have indicated the need for prophylactic aortic root surgery before dissection occurred.

AORTIC ROOT GROWTH

Mean aortic root growth was 0.5 (0.6) $\mathrm{mm} /$ year in the "uncomplicated" group (group A), which was significantly different from aortic root growth in the prophylactically operated group (group B) $(\mathrm{p}<0.0001)$ and in the dissected group (group C) $(p<0.01)$. The aortic root growth rates in groups $\mathrm{B}$ and $\mathrm{C}$ are shown in table 2 .

\section{CURRENT GUIDELINES}

In the 13 patients who developed aortic root dissection, the current guidelines for prophylactic aortic root replacement were retrospectively applied in the period preceding dissection (fig 3). In five of the 13 patients, aortic root dissection occurred at a diameter $\geqslant 55$ $\mathrm{mm}$ ( 55 to $65 \mathrm{~mm}$ ). Of the remaining eight patients, four developed aortic root dissection at a diameter of 50 to $55 \mathrm{~mm}$ with a family history of aortic dissection, and two showed excessive growth of the aortic root $(\geqslant 2$ $\mathrm{mm} /$ year), presently an indication for prophylactic replacement of the aortic root in our institution. Consequently, only two patients remained in whom, theoretically, aortic root dissection could not have been prevented by the application of the current guidelines. In neither of these two patients was any known risk factor for aortic root dissection present (other than the diagnosis Marfan's syndrome itself). The aortic root diameter of one patient had been $37 \mathrm{~mm}$ and of the other $52 \mathrm{~mm}$ for several years at the time dissection occurred. In both cases the family history was positive for Marfan's syndrome but not for aortic dissection. 


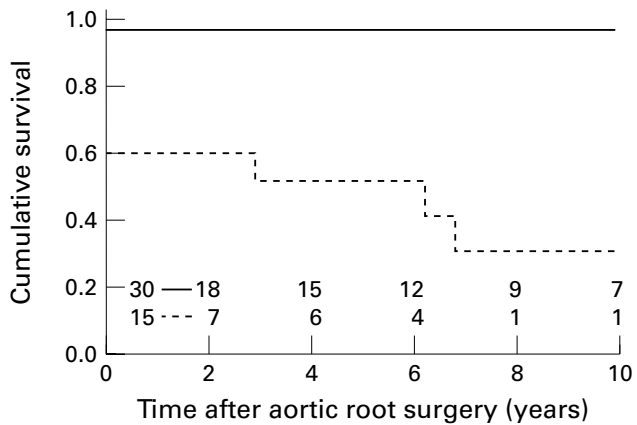

Figure 4 Kaplan-Meier survival curves of 30 patients who underwent prophylactic replacement of the aortic root and 15 patients who underwent emergency replacement of the aortic root. The numbers of patients during follow up are indicated in the lower part of the figure. A significant difference between the survival curves is demonstrated $(p<0.0001)$.

COMPARISON OF SURVIVAL AFTER PROPHYLACTIC AND EMERGENCY SURGERY

Figure 4 shows the differences in survival of patients with Marfan's syndrome after prophylactic $(n=30)$ and emergency $(n=15)$ aortic root replacement, with a five year cumulative survival of $97 \%$ and $51 \%$, respectively $(\mathrm{p}<0.001)$. Mean age during prophylactic repair did not differ significantly from the mean age during aortic root dissection, at 28 (12) $v$ 34 (14) years; $p=0.14$. Of the 30 patients who underwent prophylactic repair, one died of cardiac arrest postoperatively at the age of 12 years, one had a reoperation of the aortic root, and two underwent surgery on the descending aorta. Nine of the 15 patients (60\%) who underwent emergency repair eventually died: six died perioperatively and three died several years after emergency surgery (two following complications in the aortic arch or descending aorta, and one following complications in the aortic root). Of the six remaining patients, three developed complications in the aortic arch and descending aorta, requiring surgery.

\section{Discussion}

SURVIVAL AND EVENT FREE SURVIVAL

Our aim in this study was to determine survival and event free survival in our Marfan patient population. Five and 10 year cumulative survival following diagnosis was $95 \%$ and $88 \%$, respectively. Although long term prognosis seems reasonably good, it should be kept in mind that these figures relate to a rather young patient population (with a mean age of 21 years in our study), and that survival is far from uncomplicated in a considerable proportion of the patients. Our study showed that $34 \%$ of all diagnosed cases (43 of 125) will develop serious cardiovascular complications (varying from major cardiac surgery to death) in the following 10 years. All these findings are consistent with the results of other recent studies. $^{4-5} 1016$ Cardiovascular complications were the only cause of death, with aortic root dissection counting for $82 \%$ of overall mortality in our study. These numbers are in line with previous studies, reporting values between $71 \%$ and $100 \%$ for cardiovascular deaths, of which $80-100 \%$ are the result of aortic root dissection..$^{3-516}$
COMPARISON OF SURVIVAL AFTER PROPHYLACTIC AND EMERGENCY SURGERY

Thirty of 125 patients (24\%) underwent prophylactic surgery of the aortic root. Five year survival, which remained $97 \%$ after 10 years in these patients, was significantly better than in those undergoing emergency surgery for aortic root dissection $(51 \%$ at five years and $31 \%$ at 10 years, respectively). These findings emphasise once again the clinical importance of early intervention in patients at risk for aortic root dissection. ${ }^{28}$

\section{CURRENT GUIDELINES}

Current recently revised clinical guidelines could in theory have prevented 11 of 13 aortic root dissections ( $85 \%$ ) by prophylactic replacement of the aortic root, which seems to be a reasonable improvement over the clinical strategy of the past 14 years. Further lowering of the threshold - for example, from 55 to $50 \mathrm{~mm}$, routinely-would have prevented only one more dissection, which does not seem to outweigh the disadvantages of prophylactic surgery in a considerable number of patients. Two patients developed aortic root dissection without any predisposing risk factors, even according to current guidelines. Aortic dissection with only moderate aortic dilatation has been observed in various Marfan studies. $^{8}{ }^{17}$ 29-31 Therefore, investigation of other risk factors, such as aortic stiffness, remains important. ${ }^{32} 33$

RISK ASSESSMENT

Excessive aortic root growth has undoubtedly contributed to the decision to perform prophylactic surgery in the past 14 years. This is reflected by the high aortic root growth rates in this prophylactically operated patient group (mean $3.6 \mathrm{~mm} /$ year). In the group without any aortic complications, this growth was significantly less (mean $0.5 \mathrm{~mm} /$ year). In this population, mean aortic root growth augmented by two standard deviations was 1.7 $\mathrm{mm}$ /year, which seems reasonably in line with the threshold for excessive aortic root growth proposed by Legget et al. ${ }^{17}$ Although significant differences in the prophylactically operated group were found, the group of patients developing aortic root dissection did not show excessive aortic growth (mean $1.8 \mathrm{~mm} /$ year). It is possible that these patients are not considered to be at risk for aortic dissection because of their only mildly accelerated aortic root growth.

In the group of patients who developed aortic root dissection, lens subluxation was less often present than in the uncomplicated and prophylactically operated patients. This phenomenon was also shown in the study by Legget et al, ${ }^{17}$ and probably reflects the diagnostic importance attached to lens subluxation as a phenotypic feature in Marfan's syndrome. Lack of this classical feature is therefore associated with delayed diagnosis and more severely affected aortas. ${ }^{34}$ Thus the age at diagnosis of dissected patients in our study population was apparently higher than in the other Marfan groups. Aortic root dilatation at the time of 
dissection, however, was less pronounced than in the group who underwent prophylactic repair (at the time of surgery). A possible explanation for this observation is that 12 of the 30 patients $(40 \%)$ with prophylactic repair presented at the first visit with a severely dilated aortic root (52 to $100 \mathrm{~mm}$ ) and underwent surgery soon afterwards. Hence, mean aortic root diameter in this group exceeded the mean diameter in the dissected patients. Absence of lens subluxation as a clinical feature, and high mutation frequencies (29\% in our study, $44 \%$ in the study by Legget et $\mathrm{al}^{17}$ ), may necessitate a more aggressive screening policy to prevent cardiovascular catastrophes in a relatively young patient population.

Relative longevity in prophylactically operated Marfan patients may unmask progressive degenerative disease of the remaining aorta, resulting in aneurysmal dilatation, dissection, and rupture of the aortic arch and descending aorta, which might influence survival. ${ }^{35}{ }^{36}$ In our study, this was especially the case in the patients who underwent emergency surgery (four of five surviving patients), but also for the other patient groups. Evaluation of the entire aorta by computed tomography or magnetic resonance imaging has therefore become a crucial issue in the clinical follow up of Marfan patients, both before and after aortic root surgery.

\section{STUDY LIMITATIONS}

Our data relate only to clinically recognised Marfan patients. These patients occasionally withdrew from follow up, resulting in prophylactic surgery at a larger aortic root diameter than desired by the attending physician, or resulting in aortic root dissection.

The occurrence of lumbosacral dural ectasia, which was recently recognised as a major feature of Marfan's syndrome, ${ }^{25} 37$ was not evaluated, because these data were lacking in most patients.

Although it has been recommended that aortic root growth should be expressed in aortic ratios (relative to expected aortic root size from body surface area nomograms), ${ }^{13}{ }^{17} 19-21$ only patients older than 16 years were used for aortic root growth analysis, and physiological growth is not expected to make an important contribution to increases in aortic root diameter after that age. ${ }^{20}{ }^{21}$ The normal average aortic root growth after the age of 20 years is approximately $0.85 \mathrm{~mm} /$ decade which seems unlikely to influence our measurements. ${ }^{38}$

The influence of $\beta$ adrenergic blocking agents on aortic root growth, aortic complications, and survival was not analysed. Many of our patients were diagnosed before the beneficial effects of $\beta$ adrenergic blocking agents were known and several received treatment for only a limited period of time, or were treated with several types or dosages.

\section{CONCLUSIONS}

Survival in Marfan's syndrome seems satisfactory, although it is not complication free in many patients.
The recent revision of clinical guidelines will probably lead to a substantial reduction in aortic root dissections in patients with Marfan's syndrome. However, a few patients will develop aortic root dissection in the absence of any known risk factor. Further improvement in prognosis of Marfan patients might be achieved by the earlier detection of Marfan patients, by the application of current clinical guidelines, by evaluation and follow up investigation of the entire aorta, and by investigation of other risk factors for aortic dissection.

MG is supported by a grant from the SORBO Heart Foundation.

1 Pyeritz RE, McKusick VA. The Marfan syndrome: diagnosis and management. N Engl f Med 1979;300:772-7.

2 McKusick VA. The cardiovascular aspects of Marfan's syndrome: a heritable disorder of connective tissue. Syndrome: a heritable dis

3 Murdoch JL, Walker BA, Halpern Bl, et al. Life expectancy and causes of death in the Marfan syndrome. $N$ Engl F Med 1972;286:804-8.

4 Marsalese DL, Moodie DS, Vacante M, et al. Marfan's syndrome: natural history and long-term follow-up of cardiovascular involvement. f Am Coll Cardiol 1989;14: $422-8$.

5 Silverman DI, Burton KJ, Gray J, et al. Life expectancy in the Marfan syndrome. Am f Cardiol 1995;75:157-60.

6 Roman MJ, Devereux RB, Kramer-Fox R, et al. Prognostic significance of the pattern of aortic root dilation in the Marfan syndrome. f Am Coll Cardiol 1993;22:1470-6.

7 Kouchoukos NT, Wareing TH, Murphy SF, et al. Sixteenyear experience with aortic root replacement. Results of 72 operations. Ann Surg 1991;214:308-20.

8 Gott VL, Pyeritz RE, Cameron DE, et al. Composite graft repair of Marfan aneurysms of the ascending aorta: results in 100 patients. Ann Thorac Surg 1991;52:38-44.

9 Bentall H, De Bono A. A technique for complete replacement of the ascending aorta. Thorax 1968;23:3389.

10 Chassignolle JF, Obadia JF. Aneurysme de l'aorte thoracique dans la maladie de Marfan. Arch Mal Coeur Vaiss 1997;90:1713-21.

11 McDonald GR, Schaff HV, Pyeritz RE, et al. Surgical management of patients with the Marfan syndrome and dilatation of the ascending aorta. F Thorac Cardiovasc Surg 1981; 81:180-6.

12 Pyeritz RE, Gott VL, McDonald GR, et al. Surgical repair of the Marfan aorta: technique, indications and complications. Fohns Hopkins Med f 1982;151:71-82.

13 Pyeritz RE. Marfan syndrome: current and future clinical and genetic manifestations of cardiovascular manifestaand genetic manifestations of cardiovascular man

14 Treasure T. Elective replacement of the aortic root in Marfan's syndrome. Br Heart f 1993;69:101-3.

15 Silverman DI, Gray J, Roman MJ, et al. Family history of severe cardiovascular disease in Marfan syndrome is associated with increased aortic diameter and decreased survival. F Am Coll Cardiol 1995;26:1062-7.

16 Hwa J, Richards JG, Huang H, et al. The natural history of aortic dilatation in Marfan syndrome. Med $\mathcal{f}$ Aust 1993; 158:558-62.

17 Legget ME, Unger TA, O'Sullivan CK, et al. Aortic root complications in Marfan's syndrome: identification of a lower risk group. Heart 1996;75:389-95.

18 el Habbal MH. Cardiovascular manifestations of Marfan's syndrome in the young. Am Heart f 1992;123:752-7.

19 Roman MJ, Rosen SE, Kramer-Fox R, et al. Two dimensional echocardiographic aortic root dimensions in normal children and adults. Am f Cardiol 1989;64: 507-12.

20 Vetter U, Mayerhofer R, Lang D, et al. The Marfan syndrome-analysis of growth and cardiovascular manifessyndrome-analysis of growth and cardic
tation. Eur f Pediatr 1990;149:452-6.

21 Salim MA, Alpert BS, Ward JC, et al. Effect of betaadrenergic blockade on aortic root rate of dilation in the Marfan syndrome. Am f Cardiol 1994;74:629-33.

22 Shores J, Berger KR, Murphy EA, et al. Progression of aortic dilatation and the benefit of long-term beta-adrenergic blockade in Marfan's syndrome. N Engl f Med 1994;330: 1335-41.

23 Groenink M, Rozendaal L, Naeff MSJ, et al. Marfan syndrome in children and adolescents: predictive and prognostic value of aortic root growth for screening for aortic complications. Heart 1998;80:163-9.

24 Beighton P, de Paepe A, Danks D, et al. International nosology of heritable disorders of connective tissue, Berlin 1986. ogy of heritable disorders of conn

25 De Paepe A, Devereux RB, Dietz HC, et al. Revised diagnostic criteria for the Marfan syndrome. Am $\mathcal{F} \mathrm{Med}$ Genet 1996;62:417-26. 
26 Rozendaal L, Groenink M, Naeff MSJ, et al. Marfan syndrome in children and adolescents: an adjusted nomogram for screening aortic root dilatation. Heart 1998 79:69-72.

27 Midulla PS, Ergin A, Galla J, et al. Three faces of the Bentall procedure. $\mathcal{F}$ Cardiac Surg 1994;9:466-81.

28 Cild AH. Marfan syndrome-current medical and genetic knowledge: how to treat and when. $\mathcal{F}$ Cardiac Surg 1997;12 $131-5$

29 Pyeritz RE. Predictors of dissection of the ascending aorta in Marfan syndrome [abstract]. Circulation 1991;84:II-351.

30 Murgatroyd F, Child A, Poloniecki J, et al. Does routine echocardiographic measurement of the aortic root diameter predict the risk of aortic dissection in the Marfan syndrome? Eur Heart 7 1991;12:410.

31 Sütch G, Jenni R, von Segesser L, et al. Predictability of aortic dissection as a function of aortic diameter. Eur Heart $\mathcal{F}$

32 Hirata K, Triposkiadis F, Sparks E, et al. The Marfan syndrome: abnormal elastic properties. 7 Am Coll Cardiol 1991;18:57-63.
33 Groenink M, de Roos A, Mulder BJM, et al. Changes in aortic distensibility and pulse wave velocity assessed with magnetic resonance imaging following beta-blocker therapy in the Marfan syndrome. Am f Cardiol 1998;82:

34 Marsalese DL, Moodie DS, Lytle BW, et al. Cystic medial necrosis of the aorta in patients without Marfan's syndrome: surgical outcome and long-term follow-up. $\mathcal{F}$ Am Coll Cardiol 1990;16:68-73.

35 Finkbohner R, Johnston D, Crawford ES, et al. Marfan syndrome. Long-term survival and complications after aortic aneurysm repair. Circulation 1995;91:728-33.

36 Bachet J, Brizard C, Goudot B, et al. Repeated surgery for recurrent dissection of the aorta. Eur f Cardiothorac Surg 1990;4:238-44.

37 Pyeritz RE, Fishman EK, Bernhardt BA, et al. Dural ectasia is a common feature of the Marfan syndrome. Am $\mathcal{F ~ H u m}$

38 Venet 1988;43:726-32. echocardiographic aortic root size. Circulation 1995;91: 734-40. 